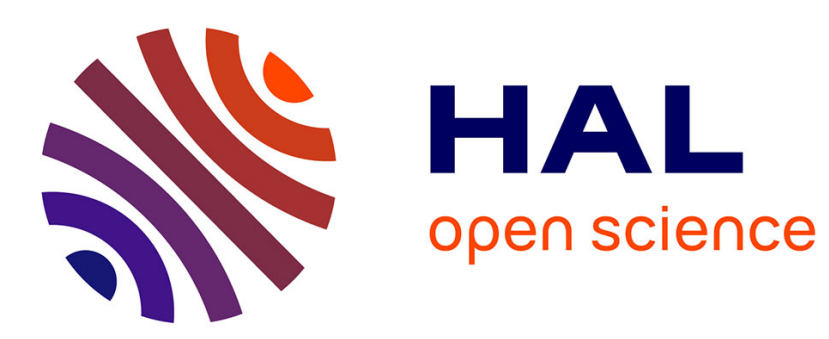

\title{
An FFT method for the computation of thermal diffusivity of porous periodic media
}

Quy-Dong To, Vincent Monchiet, Viet Thanh To

\section{To cite this version:}

Quy-Dong To, Vincent Monchiet, Viet Thanh To. An FFT method for the computation of thermal diffusivity of porous periodic media. Acta Mechanica, 2017, 228 (9), pp.3019-3037. hal-01665951

\section{HAL Id: hal-01665951 \\ https://hal.science/hal-01665951}

Submitted on 28 Mar 2021

HAL is a multi-disciplinary open access archive for the deposit and dissemination of scientific research documents, whether they are published or not. The documents may come from teaching and research institutions in France or abroad, or from public or private research centers.
L'archive ouverte pluridisciplinaire HAL, est destinée au dépôt et à la diffusion de documents scientifiques de niveau recherche, publiés ou non, émanant des établissements d'enseignement et de recherche français ou étrangers, des laboratoires publics ou privés. 


\title{
A FFT method for the Computation of thermal diffusivity of porous periodic media
}

\author{
Viet-Thanh To · Vincent Monchiet • \\ Quy Dong To
}

the date of receipt and acceptance should be inserted later

\begin{abstract}
In this paper, we provide Fast Fourier Transform iterative schemes to compute the thermal diffusivity of periodic porous medium. We consider the fluid flow through a porous rigid solid due to a prescribed macroscopic gradient of pressure and a macroscopic gradient of temperature. As already proved in the literature, the asymptotic homogenization procedure is reduced to the resolution of two separated problems for the unit cell: (i) the fluid flow governed by the Stokes equations with an applied gradient of pressure, (ii) the heat transfer by both convection and conduction due to an applied macroscopic gradient of temperature. We develop new numerical approaches based on fast Fourier transform for the implementation of the cell problems. In a first approach, a simple iterative based on the primal variable (gradient of temperature) is provided to solve the heat transfer problem. In order to improve the convergence in the range of high values of the prescribed gradient of pressure, we propose a more sophisticated iterative scheme based on the polarization. In order to evaluate their capacities, these FFT algorithms are applied to some specific microstructures of interest including flows past parallel pores (Poiseuille flows) and periodically or randomly distributed cylinders.
\end{abstract}

Keywords Porous media - Thermal diffusivity · Permeability · Fast Fourier Transform

\section{Introduction}

Studying transport processes in porous media and determining their effective behavior are problems of fundamental interest. Using the volume average $[15,24,41,42,48]$ or asymptotic homogenization $[8,9,13,46]$, a large number

Viet-Thanh To · Vincent Monchiet · Quy Dong To

Université Paris-Est, Laboratoire Modélisation et Simulation Multi Echelle, LMSME UMR8208 CNRS, 5 boulevard Descartes, 77454 Marne la Vallée Cedex, France

E-mail: vincent.monchiet@u-pem.fr 
of macroscopic models have been derived to capture successfully the phenomena. For periodic media, effective properties can generally be proved to exist uniquely and determined by solving the periodic boundary value problem on the unit cell. Regarding the coupled fluid flow/heat transfer in porous media, there are numerous works dedicated to the resolution of flow/thermal diffusion in porous media. Standard numerical methods based on Finite Element Method (FEM), Finite Volume Method (FVM) or Boundary element Methods (BEM) have been often considered for the problem of Stokes flow in porous media. For instance, $[2,3,12,17,25]$ determined the effective permeability for fluid flow past a regular array of cylinders. The computation of permeability for 3D microstructures has been performed by [11] in the case of an array of sphere and more recently by [30] on a real media imaged by microtomography. The resolution with the FEM of the Stokes flow problems requires special care, since classical interpolation based on piecewise linear function fails to perform due to the flow incompressibility. In such situation, more sophisticated interpolation known as "MINI element" must be used $[6,7,19]$. Thermal dispersion in convective flow in porous media has been numerically investigated for $2 \mathrm{D}$ problems constituted of regular arrays of cylinders, squared or elliptic rods $[5,28,35,45,47]$.

More recently, a new numerical method based on the fast Fourier Transform (FFT) has been proposed to numerically evaluate the effective permeability of porous media $[29,38,43]$ on the basis of the method first introduced by Moulinec et al. [39] for elastic composites. The local cell problem for the fluid flow is solved by means of an iterative scheme which uses the periodic Green's tensor and the images of the microstructures (which come from imagerie technics for instance). The method has significantly increased performance since the memory needed for solving the problem is greatly reduced compared to other methods.

In this paper we provide new FFT based iterative schemes to handle the problem of thermal diffusion in porous media. In a first approach, we propose an iterative formulated with the gradient of temperature. However, due to the presence of the convective term, the rate of convergence is greatly reduced at large values of the applied pressure gradient. This motivates an alternative iterative scheme based on the polarization.

The paper is organized as follows. In section 2, we present the periodic boundary value problems and we recall the definitions for effective permeability and diffusivity. Section 3 is devoted to the implementation of cell problems with FFT algorithms. After a brief recall on the resolution of the stokes problem and the implementation of the local velocity (which appears in the convection term), we focus our attention on the heat transfer problem by both conduction and convection. In section 4, we provide some illustrations. First, we consider the simple case of flows past parallel pores which has the advantage to provide analytic expression of the conductivity which serves to evaluate the accuracy of our numerical solutions. Illustrations for 2D and 3D microstructures are also provided. Particularly, the resolution of the heat transfer problem past randomly oriented fibers is performed to show the capacity of the method. 


\section{Effective transport properties for coupled heat transfer and fluid} flow problems

We consider a periodic porous medium saturated by a homogeneous Newtonian viscous fluid with the dynamic viscosity $\eta_{f}$. Both the fluid and the skeleton are thermally conductive media with the diffusivity $k_{f}$ and $k_{s}$ respectively. By $V$, we denote the total volume of the cell, by $V_{f}$ and $V_{s}$ the volume occupied by the fluid and the solid respectively, where $V_{s}$ and $V_{f}$ are both interconnected domains. The frontier between the fluid and the solid is denoted $\Gamma$. Due to the fluid motion, the mass, momentum and energy can be transported along with the stream currents, which results in the following set of local equations:

$$
\begin{cases}\eta_{f} \Delta \boldsymbol{v}-\nabla p=0 & \text { in } V_{f} \\ \operatorname{div} \boldsymbol{v}=0 & \text { in } V_{f} \\ \boldsymbol{v}=0 & \text { on } \Gamma \\ \frac{\partial T}{\partial t}+\boldsymbol{v} \cdot \nabla T=\nabla \cdot(k \nabla T) & \text { in } V\end{cases}
$$

where $\boldsymbol{v}, p, T$ and $k$ are respectively the velocity, pressure, temperature and thermal diffusivity. In our problem, the Reynolds number is assumed to be small $R e \ll 1$ and the fluid is incompressible and stationary. In writing (1), the first three equations are valid for the fluid while the last equation is valid for both the solid and the fluid by admitting a null velocity field in the solid phase. The local diffusivity $k$ is a piecewise constant function:

$$
k=k_{s} \text { in solid phase, } \quad k=k_{f} \text { in fluid phase. }
$$

The periodic homogenization procedure is based on the existence of the two following length scales: $L$ the size of the porous medium and $l$ the size of the periodic cell. The asymptotic expansion method in terms of the ratio $\epsilon=$ $l / L \ll 1$ has been used to determine the equations corresponding to every orders of $\epsilon^{k}$. The detailed derivation of the effective permeability, diffusivity, etc... has been well documented in the literature $[4,8,9,13]$.

The macroscopic description for the fluid flow in the porous medium is given by the Darcy law:

$$
\boldsymbol{V}=-\frac{1}{\eta_{f}} \boldsymbol{B} . \boldsymbol{J}
$$

where $\boldsymbol{V}$ is the macroscopic velocity, $\boldsymbol{J}$ the macroscopic pressure gradient and $\boldsymbol{B}$ the permeability tensor.

The macroscopic equivalent description for the heat transfer is:

$$
\frac{\partial T}{\partial t}+\boldsymbol{V} \cdot \boldsymbol{E}=\operatorname{div}(\boldsymbol{K} \cdot \boldsymbol{E})
$$

where $\boldsymbol{E}$ is the macroscopic gradient of temperature and $\boldsymbol{K}$ is the effective diffusivity tensor.

Finally, to find the homogenized permeability and diffusivity tensors of the 
porous medium, we must account for the periodicity conditions prescribed for the variables $\boldsymbol{v}, T$ and $p$ when solving (1). These conditions will be given in the next. For the sake of clarity, two periodic boundary value problems will be treated separately, as described in the following:

- Stokes flow problem:

$$
\begin{cases}\eta_{f} \Delta \boldsymbol{v}-\nabla p-\boldsymbol{J}=0 & \text { in } V_{f} \\ \operatorname{div} \boldsymbol{v}=0 & \text { in } V_{f} \\ \boldsymbol{v}=0 & \text { on } \partial V_{f}\end{cases}
$$

where $\boldsymbol{v}$ and $p$ are $V$-periodic, i.e. they take the same values on two opposite sides of the unit cell. $\boldsymbol{J}$ is constant in the cell and denotes the applied macroscopic pressure gradient. Due to the linearity of the equations, the local velocity is on the form:

$$
\boldsymbol{v}=\boldsymbol{A} . \boldsymbol{J}
$$

where $\boldsymbol{A}$ is the two-order localization tensor. It components $A_{i j}$ are determined by solving Eq. (5) and by considering the component $v_{i}$ due to the applied component of the pressure gradient $J_{j}$.

The permeability tensor $\boldsymbol{B}$ is given by:

$$
\boldsymbol{B}=-\eta_{f}<\boldsymbol{A}>_{V}
$$

where $<\bullet>_{V}$ denotes the volume average of the quantity "•" over the volume of the cell.

- Heat transfer problem:

$$
\nabla \cdot[k \nabla T]=\boldsymbol{v} \cdot \nabla T-\boldsymbol{V} \cdot \boldsymbol{E}
$$

where $T-\boldsymbol{E} . \boldsymbol{x}$, in which $\boldsymbol{x}$ denotes the vector position, is $V$-periodic and $\boldsymbol{v}$ is determined from the Stokes-flow problem. In other hand, the flux $\boldsymbol{j} . \boldsymbol{n}$, with $\boldsymbol{j}=k \nabla T$ and $\boldsymbol{n}$ is the outward normal unit vector taken on the boundary of the cell, is antiperiodic that is the consequence of the thermal flux equilibrium between the cell and its neighbors.

Another approximations could be derived for the macroscopic filtration law considering the Forchheimer Law [18], Darcy-Brinkman Law [21,22] or ForchheimerBrinkman law [23]. The determination of such macroscopic law in the framework of the asymptotic homogenization theory has been the subject of many studies. The derivation of the Forchheimer or more complex non linear filtration law has been proposed by Mei and Auriault [31], Wodie and Levy [49], Giorgi [20], Skjetne and Auriault [16] or Bahloff et al. [10] by accounting for the inertial term which appears in the Navier-Stokes. Also the Brinkman equation can be derived from the asymptotic homogenization theory by accounting for the higher order terms of the series [1]. Note that the present works focus on the resolution of the Heat transfer problem and the algorithms developed 
in the present paper could be also used by considering a local velocity in the convection term which accounts for such effects.

Denoting by $\boldsymbol{e}$ the temperature gradient, its average over the volume is the macroscopic gradient of temperature $\boldsymbol{E}$ :

$$
\boldsymbol{e}=\nabla T, \quad \boldsymbol{E}=\langle\boldsymbol{e}\rangle_{V}
$$

The solution of the heat transfer problem then depends on two forcing variables, the macroscopic gradient of temperature $\boldsymbol{E}$ and the gradient of pressure $\boldsymbol{J}$ due to the presence of the convection term (see relation (6) giving the velocity as function of $\boldsymbol{J}$ ).

Due to the linearity of the problem in $\boldsymbol{E}$, there exists a uniquely zero mean function $\boldsymbol{g}$ satisfying to:

$$
T=\boldsymbol{g} \cdot \boldsymbol{E}+\bar{T}, \quad\langle\boldsymbol{g}\rangle_{V}=\mathbf{0}
$$

where $\bar{T}$ is the average temperature. Note however that the local temperature $T$ has a non linear dependence with the applied pressure gradient $\boldsymbol{J}$.

The effective diffusivity $\boldsymbol{K}$ is given by:

$$
\boldsymbol{K}=\langle k\rangle \boldsymbol{I}+\left\langle k \nabla^{T} \boldsymbol{g}\right\rangle-\langle(\boldsymbol{v}-\boldsymbol{V}) \otimes \boldsymbol{g}\rangle
$$

where $\boldsymbol{I}$ is the identity for two order tensors. Note that the above definition for the diffusivity tensor is non symmetric due to the presence of the dispersive term $\langle(\boldsymbol{v}-\boldsymbol{V}) \otimes \boldsymbol{g}\rangle$. However, the permeability tensor $\boldsymbol{K}$ is defined merely by its inner product with the symmetric tensor $\nabla \boldsymbol{E}$ in relation (4), so that only the symmetric part $\boldsymbol{K}^{s}$ of $\boldsymbol{K}$ has a contribution at the macroscopic scale. Note also that, still due to the presence of this convection term, the diffusion tensor $\boldsymbol{K}$ introduces a dependence with the macroscopic pressure gradient $\boldsymbol{J}$.

The determination of the diffusivity then requires successively the resolution of the Stokes flow problem and then the heat transfer problem. Since the two problems are periodic, it is convenient to work with Fourier series and the associated FFT technique. Considering a parallelepipedic unit cell $V$, a $V$-periodic function $\phi$ admits the following representation:

$$
\phi(\boldsymbol{x})=\sum_{n} \widehat{\phi}\left(\boldsymbol{\xi}_{n}\right) \exp \left(i \boldsymbol{\xi}_{n} \cdot \boldsymbol{x}\right)
$$

where $i^{2}=-1$ and $\widehat{\phi}(\boldsymbol{\xi})$ denotes the Fourier transform of $\phi(\boldsymbol{x})$ given by:

$$
\widehat{\phi}(\boldsymbol{\xi})=\mathcal{F}(\phi(\boldsymbol{x}))=<\phi(\boldsymbol{x}) \exp (-i \boldsymbol{\xi} \cdot \boldsymbol{x})>_{V}
$$

where $\boldsymbol{\xi}_{n}$ are the wave vectors given by:

$$
\boldsymbol{\xi}_{n}=2 \pi n \boldsymbol{\zeta}, \quad n=0, \pm 1, \ldots \pm \infty, \quad \zeta_{i}=\frac{1}{h_{i}}
$$

and where $h_{1}, h_{2}, h_{3}$ are the dimensions of the cell. In Eq. (13) and in the next, we shall use the notation $\hat{\phi}_{n}$, a quantity symbol $\phi$ associated with wave vector $\boldsymbol{\xi}_{n}$, to refer to the discrete Fourier transform of $\phi(\boldsymbol{x})$ which is associated with 
the real variable $\boldsymbol{x}$. When dealing with Fourier series representation, it is convenient to use the FFT based iterative schemes which use exact expressions of the Green operator in the Fourier space. These methods, initially introduced by Moulinec and Suquet $[39,40]$ for composite materials, are particularly adapted when dealing with unit cell elementary problems with periodic conditions.

\section{Resolution of cell problems with FFT}

\subsection{Resolution of Stokes problem}

The description of the FFT method dealing with this problem have been presented in $[38,44]$ and will be recalled briefly for completeness. Generally, we can make use of the analogy between the Stokes equations and the elasticity problem of composites to formulate the integral equation in the sense of Lippmann-Schwinger-Dyson $[14,33,34]$. However, there are some details of viscous flow problem that render the problem different from the original approach developed for elastic composites. The unit cell of the porous medium can be assimilated to an elastic composite reinforced by perfectly rigid inclusions. For such class of problems a stress formulation is more suitable since it uses the compliance that is null within the inclusion while a strain based approach uses the rigidity that is singular in the inclusion. The compliance reads:

$$
\mathbb{S}(\boldsymbol{x})=\frac{1}{2 \eta_{f}} \mathrm{I}_{f}(\boldsymbol{x}) \mathbb{K}
$$

in which the fourth order tensor $\mathbb{K}$ is defined by:

$$
\mathbb{K}=\mathbb{I}-\mathbb{J}, \quad \mathbb{J}=\frac{1}{3} \boldsymbol{I} \otimes \boldsymbol{I} .
$$

In the above equation, $\boldsymbol{I}$ is the two order identity tensor, $\mathbb{I}$ is the fourth order identity tensor. $\mathbb{J}$ and $\mathbb{K}$ are fourth order projectors. The action of $\mathbb{K}$ on a two order tensor provides its deviatoric part while the action of $\mathbb{J}$ provides the spherical part. Moreover, $\mathrm{I}_{f}(\boldsymbol{x})$ is the characteristic function of the volume $V_{f}$ given by:

$$
\mathrm{I}_{f}(\boldsymbol{x})= \begin{cases}1 & \text { in } V_{f} \\ 0 & \text { in } V_{s}\end{cases}
$$

The main difference with the problem of elastic composite lies in the forcing term, which could be represented here by the body force $f$ which takes the value:

$$
\boldsymbol{f}=-\boldsymbol{J} \text { in } V_{f}, \quad \boldsymbol{f}=\boldsymbol{F}+\boldsymbol{C} \wedge \boldsymbol{x} \text { in } V_{s} .
$$

The body force in the solid phase represents the drag force due to the flow through the rigid skeleton. $\boldsymbol{F}$ is prescribed in order to makes null the translation while $\boldsymbol{C}$ is added to makes null the rotation of the solid. $\boldsymbol{F}$ and $\boldsymbol{C}$ 
physically represents the drag force due to the fluid motion. Introducing a reference medium of compliance $\mathbb{S}^{0}$, it has been shown (see [38]) that the stress field is solution of the integral equation (written in Fourier space):

$$
\widehat{\boldsymbol{\sigma}}_{n}=\widehat{\boldsymbol{\Omega}}_{n} \cdot \widehat{\boldsymbol{f}}_{n}-\widehat{\boldsymbol{\Delta}}_{n}:\left[\widehat{\mathbb{S}}_{n} * \widehat{\boldsymbol{\sigma}}_{n}-\mathbb{S}^{0}: \widehat{\boldsymbol{\sigma}}_{n}\right]
$$

where $\widehat{\boldsymbol{\Delta}}_{n}=\widehat{\boldsymbol{\Delta}}\left(\boldsymbol{\xi}_{n}\right)$ is the Green tensor for the stress whose expression can be found in [38]. The symbol "*" represents the discrete convolution product defined by:

$$
\widehat{\mathbb{S}}_{n} * \widehat{\boldsymbol{\sigma}}_{n}=\sum_{m} \widehat{\mathbb{S}}\left(\boldsymbol{\xi}_{n}-\boldsymbol{\xi}_{m}\right): \widehat{\boldsymbol{\sigma}}\left(\boldsymbol{\xi}_{m}\right)
$$

In Eq. (19), $\widehat{\Omega}_{n}=\widehat{\Omega}\left(\boldsymbol{\xi}_{n}\right)$ where the components of the third order tensor $\widehat{\boldsymbol{\Omega}}(\boldsymbol{\xi})$ are:

$$
\widehat{\Omega}_{i j k}(\boldsymbol{\xi})=\frac{i}{|\xi|}\left[2 \bar{\xi}_{i} \bar{\xi}_{j} \bar{\xi}_{k}-\delta_{i j} \bar{\xi}_{k}-\delta_{i k} \bar{\xi}_{j}-\delta_{j k} \bar{\xi}_{i}\right], \quad \overline{\boldsymbol{\xi}}=\boldsymbol{\xi} /\|\boldsymbol{\xi}\|
$$

if $\boldsymbol{\xi} \neq 0$ but $\Omega_{i j k}(\boldsymbol{\xi})=0$ if $\boldsymbol{\xi}=0$. In Eq. (21), $\|\boldsymbol{\xi}\|$ denotes the norm of $\boldsymbol{\xi}$. The solution of the integral equation is computed with the following recurrence relation:

$$
\widehat{\boldsymbol{\sigma}}_{n}^{i+1}=\widehat{\boldsymbol{\sigma}}_{n}^{i}-\widehat{\boldsymbol{\Delta}}_{n}:\left[\widehat{\mathbb{S}}_{n} * \widehat{\boldsymbol{\sigma}}_{n}^{i}\right]
$$

which starts from the following first term:

$$
\widehat{\boldsymbol{\sigma}}_{n}^{1}=\boldsymbol{\Omega}_{n} \cdot \widehat{\boldsymbol{f}}_{n}
$$

At convergence of the iterative scheme, we compute the strain rate $\widehat{\boldsymbol{d}}_{n}$ :

$$
\widehat{\boldsymbol{d}}_{n}=\widehat{\mathbb{S}}_{n} * \widehat{\boldsymbol{\sigma}}_{n}^{i}
$$

and then the velocity field $\widehat{\boldsymbol{v}}_{n}$, obtained by the integration of the strain rate in the Fourier space:

$$
\widehat{\boldsymbol{v}}_{n}=-\frac{2 i}{\left|\boldsymbol{\xi}_{n}\right|^{2}} \widehat{\boldsymbol{d}}_{n} \cdot \boldsymbol{\xi}_{n} \quad \forall \boldsymbol{\xi} \neq 0
$$

The velocity field is defined by its Fourier coefficients for all values of $\boldsymbol{\xi}_{n}$ except for $n=0$. It means that the velocity field is defined up to an added constant which represents the mean velocity field $\boldsymbol{V}$. The latter is identified by the condition $\boldsymbol{v}=0$ in the solid phase. 
3.2 Resolution of heat transfer problem

\subsubsection{Preliminaries}

Consider a field $\boldsymbol{j}$ in real space which is in equilibrium with a source term $q$ :

$$
\nabla \cdot j+q=0
$$

and a curl free field $\boldsymbol{e}$ :

$$
\nabla \wedge e=0 .
$$

We denote by $\widehat{\boldsymbol{e}}_{n}, \widehat{\boldsymbol{j}}_{n}$ and $\widehat{q}_{n}$ the Fourier coefficient of $\boldsymbol{e}, \boldsymbol{j}$ and $q$ respectively. To read the conditions (26) and (27) in Fourier space, it is convenient to introduce the projectors $\boldsymbol{P}_{n}, \boldsymbol{Q}_{n}$ and the vector $\boldsymbol{\omega}_{n}$ defined, for $n \neq 0$, by:

$$
\boldsymbol{P}_{n}=\frac{\boldsymbol{\xi}_{n} \otimes \boldsymbol{\xi}_{n}}{\left\|\boldsymbol{\xi}_{n}\right\|^{2}}, \quad \boldsymbol{Q}_{n}=\boldsymbol{I}-\boldsymbol{P}_{n}, \quad \boldsymbol{\omega}_{n}=-i \frac{\boldsymbol{\xi}_{n}}{\left\|\boldsymbol{\xi}_{n}\right\|^{2}}
$$

and:

$$
\boldsymbol{P}_{0}=\boldsymbol{Q}_{0}=0, \quad \boldsymbol{\omega}_{0}=0 .
$$

$\widehat{\boldsymbol{P}}_{n}$ and $\widehat{\boldsymbol{Q}}_{n}$ are two projectors introduced by Milton [34]. Tensor $\widehat{\boldsymbol{\omega}}_{n}$ is also considered due to the presence of the source term in (26). In the Fourier space, equations (26) and (27) read:

$$
\widehat{\boldsymbol{P}}_{n} \cdot \widehat{\boldsymbol{j}}_{n}+\widehat{\boldsymbol{\omega}}_{n} \widehat{q}_{n}=0, \quad \widehat{\boldsymbol{Q}}_{n} \cdot \widehat{\boldsymbol{e}}_{n}=0 .
$$

When the last condition is verified, $\boldsymbol{e}$ derives from the temperature $T$, whose Fourier coefficients are obtained from:

$$
\widehat{T}_{n}=\widehat{\boldsymbol{\omega}}_{n} \cdot \widehat{\boldsymbol{e}}_{n} .
$$

In the next, two FFT based iterative schemes are proposed to solve the heat transfer problem.

\subsubsection{A primal iterative scheme}

In this section we provide a simple iterative scheme to compute the solution of the heat transfer problem. It is formulated with the primal variable: the gradient of temperature $\boldsymbol{e}$ (the associated dual variable being the flux $\boldsymbol{j}$ ). Introducing in Eq. (8) a reference medium of diffusivity $k_{0}$, it leads to:

$$
\nabla \cdot\left(k_{0} \nabla T+\tau\right)+q=0
$$

where we have introduced:

$$
\boldsymbol{\tau}=\left(k-k_{0}\right) \nabla T, \quad q=\boldsymbol{V} . \boldsymbol{E}-\boldsymbol{v} . \nabla T .
$$

Eq. (32) can be also read in Fourier space (using Eq. (30)):

$$
\widehat{\boldsymbol{P}}_{n} \cdot\left(k_{0} \widehat{\boldsymbol{e}}_{n}+\widehat{\boldsymbol{\tau}}_{n}\right)+\widehat{\boldsymbol{\omega}}_{n} \widehat{q}_{n}=0, \quad \widehat{\boldsymbol{Q}}_{n} \cdot \widehat{\boldsymbol{e}}_{n}=0 .
$$


The first relation expresses the equilibrium of the flux with the applied polarization $\tau$ and the source term $q$. The second relation is the compatibility for the gradient of temperature $\boldsymbol{e}$.

Considering that $\widehat{\boldsymbol{\tau}}_{n}$ and $\widehat{q}_{n}$ are applied to the system, the solution for $\widehat{\boldsymbol{e}}_{n}$ is:

$$
\widehat{\boldsymbol{e}}_{n}=-\widehat{\boldsymbol{G}}_{n}^{0} \cdot\left(\widehat{\boldsymbol{\tau}}_{n}+\widehat{\boldsymbol{\omega}}_{n} \widehat{q}_{n}\right) \text { for } n \neq 0, \widehat{\boldsymbol{e}}_{0}=\boldsymbol{E}
$$

where:

$$
\widehat{\boldsymbol{G}}_{n}^{0}=\frac{1}{k_{0}} \widehat{\boldsymbol{P}}_{n}
$$

is the Green function of the reference medium of diffusivity $k_{0}$.

Replacing $\widehat{\boldsymbol{\tau}}_{n}$ and $\widehat{q}_{n}$ by their expressions as function of $\widehat{\boldsymbol{e}}_{n}$ we obtain:

$$
\widehat{\boldsymbol{e}}_{n}=-\widehat{\boldsymbol{G}}_{n}^{0} \cdot\left(\widehat{k}_{n} * \widehat{\boldsymbol{e}}_{n}-k_{0} \widehat{\boldsymbol{e}}_{n}-\widehat{\boldsymbol{\omega}}_{n}\left(\widehat{\boldsymbol{v}}_{n} * \widehat{\boldsymbol{e}}_{n}\right)\right) \text { for } n \neq 0, \widehat{\boldsymbol{e}}_{0}=\boldsymbol{E}
$$

where the definition for the discrete convolution product "*" has been already given in Eq. (20). The solution of this equation is expanded along Neumann series, each term of this series being computed from the following iterative scheme:

$$
\widehat{\boldsymbol{e}}_{n}^{i+1}=-\widehat{\boldsymbol{G}}_{n}^{0} \cdot\left(\widehat{k}_{n} * \widehat{\boldsymbol{e}}_{n}^{i}-k_{0} \widehat{\boldsymbol{e}}_{n}^{i}-\widehat{\boldsymbol{\omega}}_{n}\left(\widehat{\boldsymbol{v}}_{n} * \widehat{\boldsymbol{e}}_{n}^{i}\right)\right) \text { for } n \neq 0, \quad \widehat{\boldsymbol{e}}_{0}^{i+1}=\boldsymbol{E} .
$$

A simplification of the above iterative scheme is possible by accounting that for any curl free field $e$ we have:

$$
\forall n \neq 0 \quad \widehat{\boldsymbol{G}}_{n}^{0} \cdot\left(k_{0} \widehat{\boldsymbol{e}}_{n}^{i}\right)=\widehat{\boldsymbol{e}}_{n}^{i}
$$

and

$$
\widehat{\boldsymbol{G}}_{n}^{0} \cdot\left(k_{0} \widehat{\boldsymbol{e}}_{n}^{i}\right)=0
$$

if $n=0$.

Using this simplification, we obtain for the iterative scheme:

$$
\widehat{\boldsymbol{e}}_{n}^{i+1}=\widehat{\boldsymbol{e}}_{n}^{i}-\widehat{\boldsymbol{G}}_{n}^{0} \cdot\left(\widehat{k}_{n} * \widehat{\boldsymbol{e}}_{n}^{i}-\widehat{\boldsymbol{\omega}}_{n}\left(\widehat{\boldsymbol{v}}_{n} * \widehat{\boldsymbol{e}}_{n}^{i}\right)\right)
$$

and which starts from the first term:

$$
\widehat{\boldsymbol{e}}_{n}^{1}=0 \text { for } n \neq 0, \quad \widehat{\boldsymbol{e}}_{0}^{1}=\boldsymbol{E} .
$$

At convergence, one can compute the temperature by:

$$
\widehat{T}_{n}=\widehat{\boldsymbol{\omega}}_{n} \cdot \widehat{\boldsymbol{e}}_{n}
$$

that leadings to $\boldsymbol{g}$ and then the effective diffusivity (see Eq. (10) and Eq. (11)). In Eq. (41), the convolution product between $\widehat{k}_{n}$ and $\widehat{\boldsymbol{e}}_{n}^{i}$, also $\widehat{\boldsymbol{v}}_{n}$ and $\widehat{\boldsymbol{e}}_{n}^{i}$ are effected by means of the FFT algorithm. This means that one inverse FFT and two FFT are needed at each iteration. One inverse FFT is needed to compute $\widehat{\boldsymbol{e}}_{n}^{i}$ in real space. Therefore, the product of $\boldsymbol{e}_{n}$ with $k_{n}$ and $\boldsymbol{v}_{n}$ is made in real space, in which the product is local. Thereafter two FFT are applied to compute $k \boldsymbol{e}$ and $\boldsymbol{v} . \boldsymbol{e}$ in Fourier space and to apply the product with the Green tensor. 
In (41) the velocity field $\boldsymbol{v}$ is computed by solving the Stokes problem for which the stress based iterative scheme (22) is used together with (24) and (25). The velocity field being known in Fourier space from (25), the inverse FFT is taken to obtain $\boldsymbol{v}$ in real space and is thereafter stored to make the product with $\boldsymbol{e}$ at each iteration in (41).

The details about the numerical integration of the algorithm are given below:

\begin{tabular}{ll}
\hline Initialization: & $\widehat{\boldsymbol{e}}_{n}^{1}=0$ for $n \neq 0, \widehat{\boldsymbol{e}}_{0}^{1}=\boldsymbol{E}$ \\
Iteration: & $\widehat{\boldsymbol{e}}_{n}^{i}$ is known \\
& $\boldsymbol{e}_{n}^{i}=\mathrm{FFT}^{-1}\left(\widehat{\boldsymbol{e}}_{n}^{i}\right)$ \\
& $\boldsymbol{j}_{n}=k_{n} \boldsymbol{e}_{n}^{i}$ \\
& $q_{n}=-\boldsymbol{v}_{n} \cdot \boldsymbol{e}_{n}^{i}$ \\
& $\widehat{\boldsymbol{j}}_{n}=\operatorname{FFT}\left(\boldsymbol{j}_{n}\right)$ \\
& $\widehat{q}_{n}=\operatorname{FFT}\left(q_{n}\right)$ \\
& $\widehat{\boldsymbol{e}}_{n}^{i+1}=\widehat{\boldsymbol{e}}_{n}^{i}-\widehat{\boldsymbol{G}}_{n}^{0} \cdot\left(\widehat{\boldsymbol{j}}_{n}+\widehat{\boldsymbol{\omega}}_{n} \widehat{q}_{n}\right)$ \\
\hline
\end{tabular}

The iterative scheme is stopped when the convergence criterion is satisfied. This criterion is based on the equilibrium of the flux:

$$
\left[\sum_{n}\left\|\widehat{\boldsymbol{P}}_{n} \cdot \widehat{\boldsymbol{j}}_{n}+\widehat{\boldsymbol{\omega}}_{n} \widehat{q}_{n}\right\|^{2}\right]^{1 / 2}<\epsilon
$$

where $\epsilon$ is the precision chosen equal to $10^{-3}$ in our calculations. This criterion represents a measure of the residue of the linear equation which have to be solved.

As shown in $[32,36]$ for the case of elastic composite or [37] for the case of conductivity, basic schemes based on primal or dual variables encounter convergence rate problems at high contrast. Furthermore, with the presence of the convection term in the integral equations, the basic scheme may also diverge by increasing the value of pressure gradient. Indeed, the convergence is ensured if all the eigenvalues of the linear operator giving $\widehat{\boldsymbol{e}}_{n}^{i+1}$ as function of $\widehat{\boldsymbol{e}}_{n}^{i}$ in Eq. (41) are comprised between -1 and 1. The recurrence relation (41) can be put into the form:

$$
\widehat{\boldsymbol{e}}_{n}^{i+1}=\sum_{m}\left[\delta_{m n} \boldsymbol{I}-\widehat{\boldsymbol{G}}_{n}^{0} \cdot \boldsymbol{Z}_{n m}\right] \cdot \widehat{\boldsymbol{e}}_{m}^{i}
$$

where

$$
\boldsymbol{Z}_{n m}=\widehat{k}\left(\boldsymbol{\xi}_{n}-\boldsymbol{\xi}_{m}\right) \boldsymbol{I}-\widehat{\boldsymbol{\omega}}_{n} \otimes \widehat{\boldsymbol{v}}\left(\boldsymbol{\xi}_{n}-\boldsymbol{\xi}_{m}\right)
$$

and $\delta_{m n}$ is the Kronecker symbol. Since $\widehat{\boldsymbol{e}}_{n}^{i}$ is compatible, it is invariant by the transformation $\widehat{\boldsymbol{e}}_{n}^{i} \rightarrow \boldsymbol{P}_{n} . \widehat{\boldsymbol{e}}_{n}^{i}$ for any $n \neq 0$. Moreover, the Green operator $\widehat{\boldsymbol{G}}_{n}^{0}$ is proportional to the projector $\boldsymbol{P}_{n}$ with the constant $1 / k_{0}$. It follows that Eq. (46) can be rewritten into the equivalent form:

$$
\left\{\begin{array}{l}
\widehat{\boldsymbol{e}}_{n}^{i+1}=\boldsymbol{P}_{n} \cdot \sum_{m}\left[\delta_{n m} \boldsymbol{I}-\frac{1}{k_{0}} \boldsymbol{Z}_{n m}\right] \widehat{\boldsymbol{e}}_{m}^{i} \forall n \neq 0 \\
\widehat{\boldsymbol{e}}_{0}^{i+1}=\widehat{\boldsymbol{e}}_{0}^{i}
\end{array}\right.
$$


A sufficient condition for having the convergence of the iterative scheme is that the operator

$$
\delta_{n m} \boldsymbol{I}-\frac{1}{k_{0}} \boldsymbol{Z}_{n m}
$$

has a norm less than 1 . In the case of conductive phase (the convection term being zero), the convergence has been already examined in [34]. It has been proved that the above condition is ensured for:

$$
k_{0}>\frac{1}{2} k_{\max }
$$

where $k_{\max }$ is the higher value taken by the diffusivity (here the higher value between the diffusivity of the fluid and of the solid). The following value for $k_{0}$ can be chosen in order to obtain the better rate of convergence:

$$
k_{0}=\frac{1}{2}\left(k_{f}+k_{s}\right)
$$

where $k_{f}$ and $k_{s}$ denote the diffusivity of the fluid and of the solid respectively. The rate of convergence decreases by increasing the contrast (defined as the ratio between the diffusivity of the phases) and for infinity values of the contrast the iterative scheme diverges. For the dispersive heat transfer problem, the derivation of convergence conditions is more difficult due to the presence of the velocity which depends on the microstructure geometry. Obviously, the eigenvalues of the operator $\boldsymbol{Z}_{n m}$ could be evaluated numerically. However the computation of $\boldsymbol{Z}_{n m}$ is very long and memory costly (particularly for high dimension problems such as 3D microstructures). In (44), this operator is not computed since the product between $\boldsymbol{Z}_{n m}$ and $\widehat{\boldsymbol{e}}_{m}^{i}$ is effected in the real space in which this product becomes local.

Despite this incapacity to obtain simple convergence conditions, the optimal reference modulus $k_{0}$ can be determined numerically on a lower grid. Note however that the velocity field, which enters in the recurrence relation, linearly depends on the pressure gradient. This suggests that the case of high values of the gradient of pressure is comparable to that of high values of the contrast for which the iterative scheme leads to large number of iterations. To improve the convergence issue, we propose in the next section a more sophisticated iterative scheme based on the polarization.

\subsubsection{A polarization based iterative scheme}

A polarization based iterative scheme has been provided in $[36,37]$ in the case of elastic composites. It improves the convergence of simple iterative schemes based on the strain or stress in the domain of high contrasts. In this section we propose to adapt this approach for the heat transfer problem. To this end, let us first introduce the polarization:

$$
\boldsymbol{\tau}=\left(k-k_{0}\right) \boldsymbol{e} .
$$


It follows that the gradient of temperature and the flux are given by:

$$
\boldsymbol{e}=\left(k-k_{0}\right)^{-1} \boldsymbol{\tau}, \boldsymbol{j}=\boldsymbol{\tau}+k_{0} \boldsymbol{e}
$$

The equilibrium of the flux with the convection term can be read:

$$
\widehat{\boldsymbol{G}}_{n}^{0} \cdot\left[\widehat{\boldsymbol{j}}_{n}+\widehat{\omega}_{n} \widehat{q}_{n}\right]=0
$$

while the compatibility for $\boldsymbol{e}$ can be also read:

$$
\widehat{\boldsymbol{D}}_{n}^{0} \cdot \widehat{\boldsymbol{e}}_{n}=0
$$

where $\boldsymbol{D}^{0}$ is the dual Green operator whose coefficients are given, for $n \neq 0$, by:

$$
\widehat{\boldsymbol{D}}_{n}^{0}=k_{0} \widehat{\boldsymbol{Q}}_{n}
$$

where $\widehat{\boldsymbol{Q}}_{n}$ is defined in (28) and $\widehat{\boldsymbol{D}}_{n}^{0}=0$ when $n=0$. Additionally, the average of gradient of pressure must be equal to $\boldsymbol{E}$, which reads in Fourier space:

$$
\widehat{\boldsymbol{e}}_{0}=\boldsymbol{E}
$$

To summarize, we search the value of the polarization $\tau$ which comply to relations (53) with the conditions (54), (55) and (57). The polarization must then be computed with the following iterative process:

$$
\begin{array}{ll}
\hline \text { Initialization: } & \widehat{\boldsymbol{\tau}}_{n}^{1}=0 \\
\text { Iteration: } & \widehat{\boldsymbol{\tau}}_{n}^{i} \text { is known } \\
& \boldsymbol{\tau}_{n}^{i}=\mathrm{FFT}^{-1}\left(\widehat{\boldsymbol{\tau}}_{n}^{i}\right) \\
& \boldsymbol{e}_{n}^{i}=\left(k-k_{0}\right)^{-1} \boldsymbol{\tau}_{n}^{i} \\
& \boldsymbol{j}_{n}^{i}=\boldsymbol{\tau}_{n}^{i}+k_{0} \boldsymbol{e}_{n}^{i} \\
& q_{n}^{i}=-\boldsymbol{v}_{n} \cdot \boldsymbol{e}_{n}^{i} \\
& \widehat{\boldsymbol{e}}_{n}^{i}=\operatorname{FFT}\left(\boldsymbol{e}_{n}^{i}\right) \\
& \widehat{\boldsymbol{j}}_{n}^{i}=\operatorname{FFT}\left(\boldsymbol{j}_{n}^{i}\right) \\
& \widehat{q}_{n}^{i}=\operatorname{FFT}\left(q_{n}^{i}\right) \\
& \widehat{\boldsymbol{\tau}}_{n}^{i+1}= \begin{cases}\widehat{\boldsymbol{\tau}}_{n}^{i}-\alpha \boldsymbol{K}^{0} \cdot \widehat{\boldsymbol{G}}_{n}^{0} \cdot\left(\widehat{\boldsymbol{j}}_{n}^{i}+\widehat{\boldsymbol{\omega}}_{n}^{i} \widehat{q}_{n}^{i}\right), \text { for } n \neq 0 \\
+\alpha \widehat{\boldsymbol{D}}_{n}^{0} \cdot \widehat{\boldsymbol{e}}_{n}^{i} \\
\widehat{\boldsymbol{\tau}}_{0}^{i+1}=\widehat{\boldsymbol{\tau}}_{0}^{i}+\alpha \boldsymbol{K}^{0} \cdot\left(\widehat{\boldsymbol{e}}_{0}^{i}-\boldsymbol{E}\right), \text { for } n=0\end{cases} \\
\hline
\end{array}
$$

When the convergence is reached, that corresponding to $\tau_{n}^{i+1}=\tau_{n}^{i}$, the conditions (54), (55) and (57) are recovered for the flux and gradient of temperature. This new iterative scheme uses one inverse FFT and three FFT at each iteration that is one FFT more than the simple one based on the primal variable. However, it is expected to reduce significantly the number of iterations at convergence with this new algorithm. The capacity of these methods of resolutions is evaluated in the next section.

Let us now discuss about the choice of $k_{0}$ and coefficients $\alpha$. When the convection term is null (that corresponding to $\boldsymbol{J}=0$ ), the convergence of the algorithm is guaranteed if (see [37]):

$$
\alpha \in] 0,2\left[, \quad k_{0} \in\right]-\infty, 0[
$$


Considering now the convection term, the analysis of convergence encounters the same difficulties that for the simple iterative scheme and it is some not guaranteed that the conditions (59) are still valid when the convection term is added. Consequently, the optimal choice of $k_{0}$ must then be evaluated numerically.

For the accelerated scheme, three conditions are tested to stop the iterations: (i) a first for the equilibrium of the flux, (ii) a second for the compatibility of the gradient of temperature and (iii) a condition is written for the equality between the applied macroscopic gradient of temperature and the average of the local gradient of temperature.

$$
\begin{aligned}
& \text { (i) }\left[\sum_{n}\left\|\widehat{\boldsymbol{P}}_{n} \cdot \widehat{\boldsymbol{j}}_{n}+\widehat{\boldsymbol{\omega}}_{n} \widehat{q}_{n}\right\|^{2}\right]^{1 / 2}<\epsilon \\
& \text { (ii) }\left[\sum_{n}\left\|\widehat{\boldsymbol{Q}}_{n} \cdot \widehat{\boldsymbol{e}}_{n}\right\|^{2}\right]^{1 / 2}<\epsilon \\
& \text { (iii) }\left\|\widehat{\boldsymbol{e}}_{0}-\boldsymbol{E}\right\|<\epsilon
\end{aligned}
$$

Note that the conditions (ii) and (iii) are verified at any step of the primal iterative scheme. For the polarization iterative scheme the iteration is stopped when the three conditions (i), (ii) and (iii) are satisfied.

\section{Applications}

4.1 The flow between two parallel planes

We consider a benchmark problem where the porous media is constituted of parallel pores of height $h$ regularly distributed along the direction $O x_{2}$. The volume fraction of the pores is denoted $f$. The fluid flow is generated by applying the pressure gradient $J_{1}$ along the direction $O x_{1}$. An analytic solution is given in appendix A and is used to evaluate the accuracy of the FFT numerical solutions. For the numerical implementation with FFT, a squared unit cell of dimension $2 H=1$ along each space direction is considered and a rectangular pore is centered at the origin (see Fig. 1). The volume fraction is of the pores is $f=h / H$. 


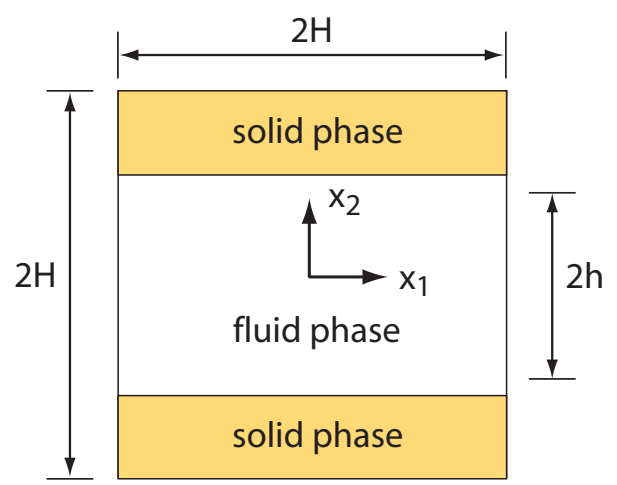

Fig. 1 Unit cell of the periodic porous solid with parallel pores.

For the applications, we put $f=0.5$ and the diffusivity contrast is $k_{f} / k_{s}=$ 10. First of all, we analyse the convergence of the two FFT based iterative schemes: the basic scheme formulated with the gradient of temperature (cf. (44)) and the accelerated scheme based on the polarization (cf. (58)). As already mentioned in the last section, the convergence of these iterative schemes depends on the contrast between the phase diffusivity but also depends on the value of the applied pressure gradient. Unfortunately, it has not been possible to derive the conditions giving the convergence for the reference diffusivity $k_{0}$, the latter must be determined numerically. Since the optimal value of $k_{0}$ is nearly independent on the number of wave vectors, it can be determined on a lower size grid. In the present example, we use $k_{0}=12$ for the basic scheme and $k_{0}=-3$ for the accelerated scheme. Also, the value $\alpha=3 / 2$ considered in [37] has been taken. With these choices, it possible to solve the thermal diffusion problem on a large range of pressure gradients. In this section and in the next parts of the paper, we work with the dimensionless pressure gradient $\boldsymbol{J}^{*}$ defined by:

$$
\boldsymbol{J}^{*}=\frac{(2 H)^{3}}{\eta_{f} k_{s}} \boldsymbol{J}
$$

On Fig. 2, we represent the number of iterations as function of the applied gradient of pressure $J_{1}^{*}$ for the basic and the accelerated scheme. Although for low values of $J_{1}^{*}$ the number of iterations at convergence is lower for the basic scheme, it can be observed that the accelerated scheme has a better rate of convergence for higher values of $J_{1}^{*}$. Also, it must be noted that the number of iterations with the basic scheme linearly increases with the applied gradient of pressure $J_{1}^{*}$ (in the log frame). However, with the accelerated scheme, the number of iterations is quasi independent of the gradient of pressure. Since it has a better rate of convergence, only the accelerated scheme is used for the applications provided in the next sections of the paper. 
Number of iterations



Fig. 2 Number of iterations at convergence of the iterative schemes as function of the applied (dimensionless) pressure gradient $J_{1}^{*}$. The volume fraction is $f=0.5$ and the contrast is $k_{f} / k_{s}=10$.

On Fig. ?? we represent the effective diffusivity $K_{11}$ (re-scaled by $k_{s}$ ) as function of the gradient of pressure $J_{1}^{*}$ obtained with the basic and accelerated scheme. The results are compared with the exact solution. Note that for the numerical implementation, $N=64$ wave vectors are considered along each space direction. Note also that the effective diffusivity $K_{11}$ displays two regimes :

- at low values of the pressure gradient, the effective diffusivity is not influenced by the pressure gradient and is equal to that computed at $J_{1}^{*}=0$.

- in the range of higher values of pressure gradient $\left(J_{1}^{*}>10^{3}\right)$, the convective term becomes predominant in the heat transfer problem. The dependence of the effective diffusivity with the applied gradient of pressure $J_{1}^{*}$ is linear in the log-log frame.

This suggests that only two points must be computed to determine the asymptotic behavior in the domain of high pressure gradient. Since the three curves are very closed on Fig. (3), a zoom is provided to display their differences. A good agreement between the exact and the numerical solution is observed. 


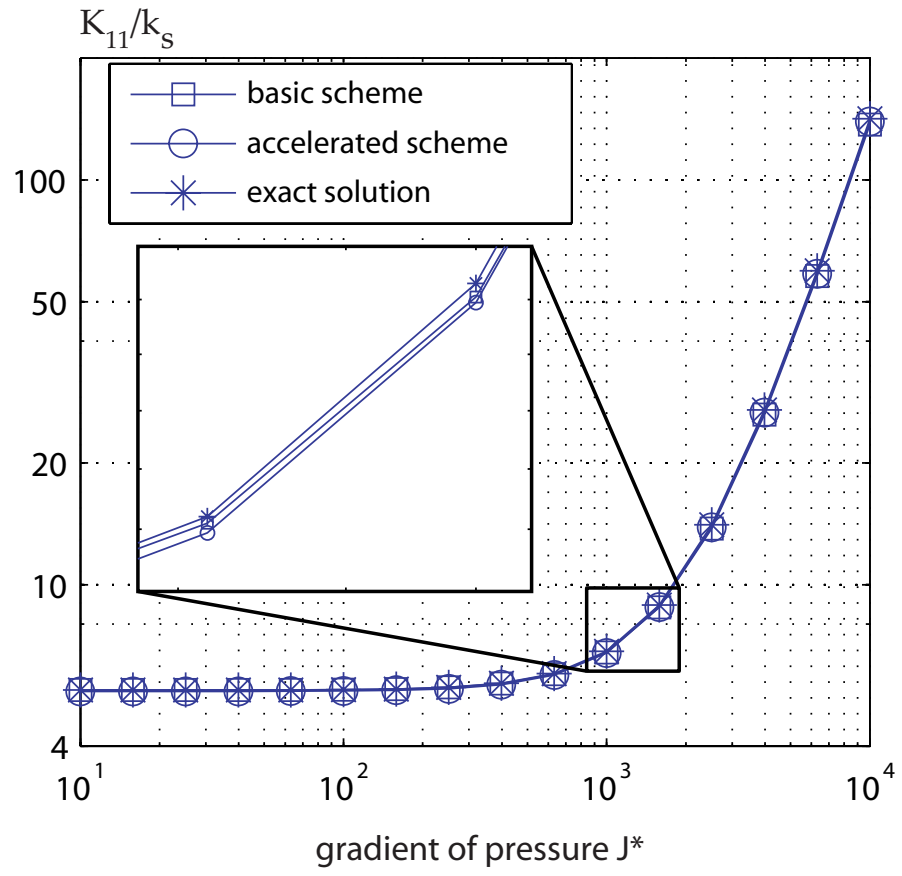

Fig. 3 Effective diffusivity of the porous medium with parallel pores. The volume fraction is $f=0.5$ and the contrast is $k_{f} / k_{s}=10$. Comparison of the FFT solutions with the exact one.

The differences observed with the exact solution can be reduced by increasing the number of wave vectors. For completeness, we then provide, on tables 1 and 2 , the values of effective diffusivity $K_{11} / k_{s}$ computed with the applied gradient of pressure $J_{1}^{*}=1000$ and $J_{1}^{*}=10000$ and for various values of the number of wave vectors. Table 1 displays the results obtained with the basic scheme while table 2 provides the same results computed with the accelerated scheme. In each table, we also provide (in the last line) the exact value of the effective diffusivity. The errors in percents, are given between the parenthesis. It can be noted that the numerical solutions converge uniformly to the exact solution by increasing the number of wave vectors.

It is observed that for the lower values of the applied pressure gradient, the primal iterative scheme gives a better estimation of the effective permeability (see table 1 and 2 for $J_{1}^{*}=10^{3}$ ) whereas at higher values of the applied pressure gradient (see 1 and 2 for $J_{1}^{*}=10^{4}$ ) the accelerated scheme provides a more accurate solutions. In the case of elasticity, a hierarchization of the FFT solutions has been proved in [?]. It has been show that the iterative schemes based on the primal or dual variables always provide a more accurate solution than that obtained with an accelerated scheme. This is also true for the heat transfer problem without the convection term. When this term is accounted in the computation of the macroscopic diffusivity, the hierarchization of the 
FFT solutions is uncertain and depends on the velue of the applied pressure gradient.

\begin{tabular}{c|cc|cc|} 
& \multicolumn{2}{|c|}{$J_{1}^{*}=1000$} & \multicolumn{2}{c|}{$J_{1}^{*}=10000$} \\
\hline $64 \times 64$ & 6.8091 & $(0.73 \%)$ & 137.0031 & $(3.1 \%)$ \\
$128 \times 128$ & 6.8305 & $(0.42 \%)$ & 139.1757 & $(1.6 \%)$ \\
$256 \times 256$ & 6.8414 & $(0.27 \%)$ & 140.2743 & $(0.83 \%)$ \\
$512 \times 512$ & 6.8468 & $(0.19 \%)$ & 140.8266 & $(0.44 \%)$ \\
\hline exact & 6.8596 & & 141.4566 &
\end{tabular}

Table 1 Effective diffusivity $K_{11} / k_{s}$ computed with the basic scheme as function of the gradient of pressure $J_{1}^{*}$ and the number of wave vectors. Solutions obtained with the simple FFT scheme.

\begin{tabular}{c|cc|cc|} 
& \multicolumn{2}{|c|}{$J_{1}^{*}=1000$} & \multicolumn{2}{c|}{$J_{1}^{*}=10000$} \\
\hline $64 \times 64$ & 6.7886 & $(1.0 \%)$ & 139.1980 & $(1.6 \%)$ \\
$128 \times 128$ & 6.8159 & $(0.64 \%)$ & 140.2881 & $(0.83 \%)$ \\
$256 \times 256$ & 6.8296 & $(0.44 \%)$ & 140.8360 & $(0.44 \%)$ \\
$512 \times 512$ & 6.8364 & $(0.34 \%)$ & 141.1106 & $(0.24 \%)$ \\
\hline exact & 6.8596 & & 141.4566 &
\end{tabular}

Table 2 Effective diffusivity $K_{11} / k_{s}$ computed with the accelerated scheme as function of the gradient of pressure $J_{1}^{*}$ and the number of wave vectors. Solutions obtained with the accelerated FFT scheme.

4.2 Flow past an array of cylinders

We now consider the flow through a periodic network of cylinders with circular cross section aligned in the direction $O x_{3}$. The radius of the cylinders is denoted $R$. For the numerical implementation of the FFT schemes, a squared unit cell of dimension $2 H=1$ and containing a circle of radius $R$ located at the origin has been considered (see figure 4). The problem is two dimensional and $N=256$ wave vectors are considered in each space directions. Also, only the accelerated scheme has been considered for this application. 


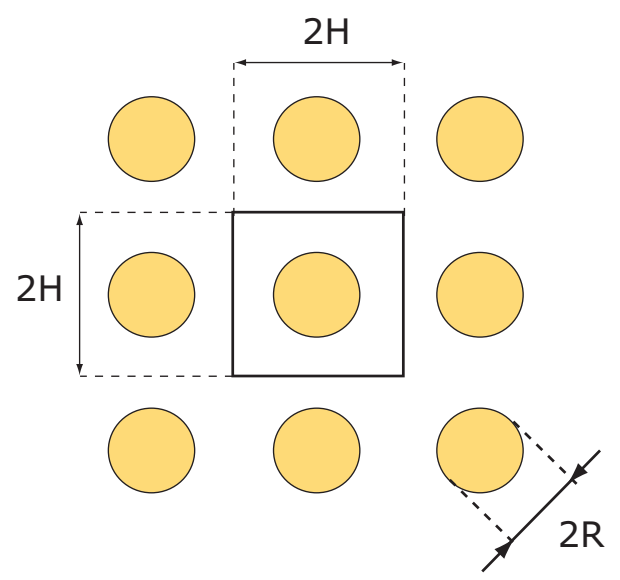

Fig. 4 Unit cell of the periodic porous solid with aligned cylinders of circular cross sections.

On Fig. 5 we represent the variations of the effective diffusivity $K_{11}$ as function of the ratio $R / H$. In this illustration, the flow is generated by imposing the gradient of pressure $J_{1}^{*}$. The contrast in the local diffusivity of the solid cylinder and the fluid is $k_{f} / k_{s}=10$ where $k_{s}$ and $k_{f}$ referred to the solid and the fluid respectively. The computation are performed by considering various values of the gradient of pressure $J_{1}^{*}$. It can be observed that, for any value of the applied pressure gradient, the effective diffusivity decreases by increasing the radius of the cylinder, since its diffusivity is lower that of the fluid. Also, the gradient of pressure increases the effective diffusivity of the porous medium. 


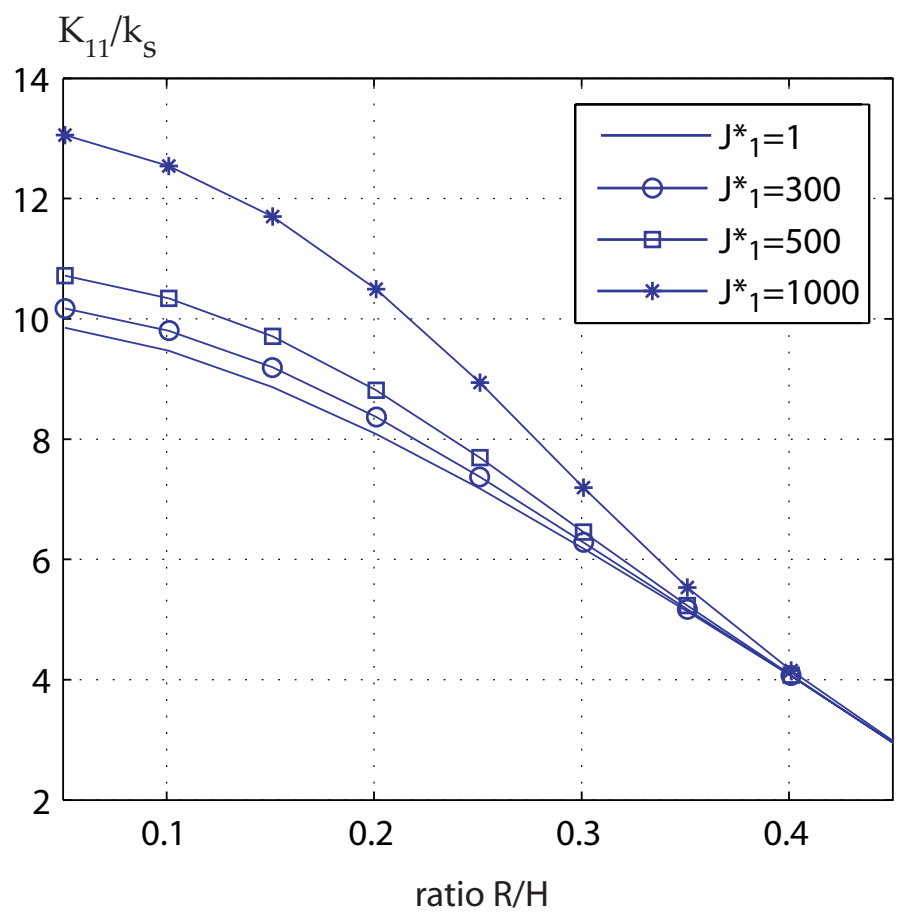

Fig. 5 Effective permeability (re-scaled by $k_{s}$ ) as function of the radius of the cylinder for a contrast $k_{f} / k_{s}=10$ and for various values of the dimensionless pressure gradient $J_{1}^{*}$.

Now, we investigate the dependence of the effective diffusivity $K_{11}$ with the direction of flow. Indeed, in the last example, the diffusivity $K_{11}$ is computed by imposing the macroscopic gradient of temperature $E_{1}$ and a gradient of pressure in the same direction. Now, the macroscopic gradient of temperature $E_{1}$ is still applied to compute $K_{11}$ but the flow is generated along different directions in the plane $O x_{1} x_{2}$. To this end, we put for the component of the gradient of pressure:

$$
J_{1}^{*}=J^{*} \cos (\theta), \quad J_{2}^{*}=J^{*} \sin (\theta)
$$

where $J^{*}$ is the norm of the applied pressure gradient $\boldsymbol{J}^{*}$ and $\theta$ lies in the range $[0,2 \pi]$. From Fig. 6 to Fig. 8 we provide the variations of the effective diffusivity with the direction of flow in a polar frame. On each figures various values of the norm of the pressure gradient $J^{*}$ have been considered. It must be observed that the diffusivity is affected by the flow direction, particularly when the flow is parallel to the applied gradient of temperature. When the gradient of pressure is applied in the direction transverse to the temperature gradient $\left(\theta=90^{\circ}\right)$ the effective diffusivity is not affected by $J^{*}$. It must be also noted that the influence of the flow direction is more important when the 
radius of the cylinder is small. For larger values of the cylinder (for instance, $R / H=0.8$ on Fig. 8 ) these effects are greatly reduced.

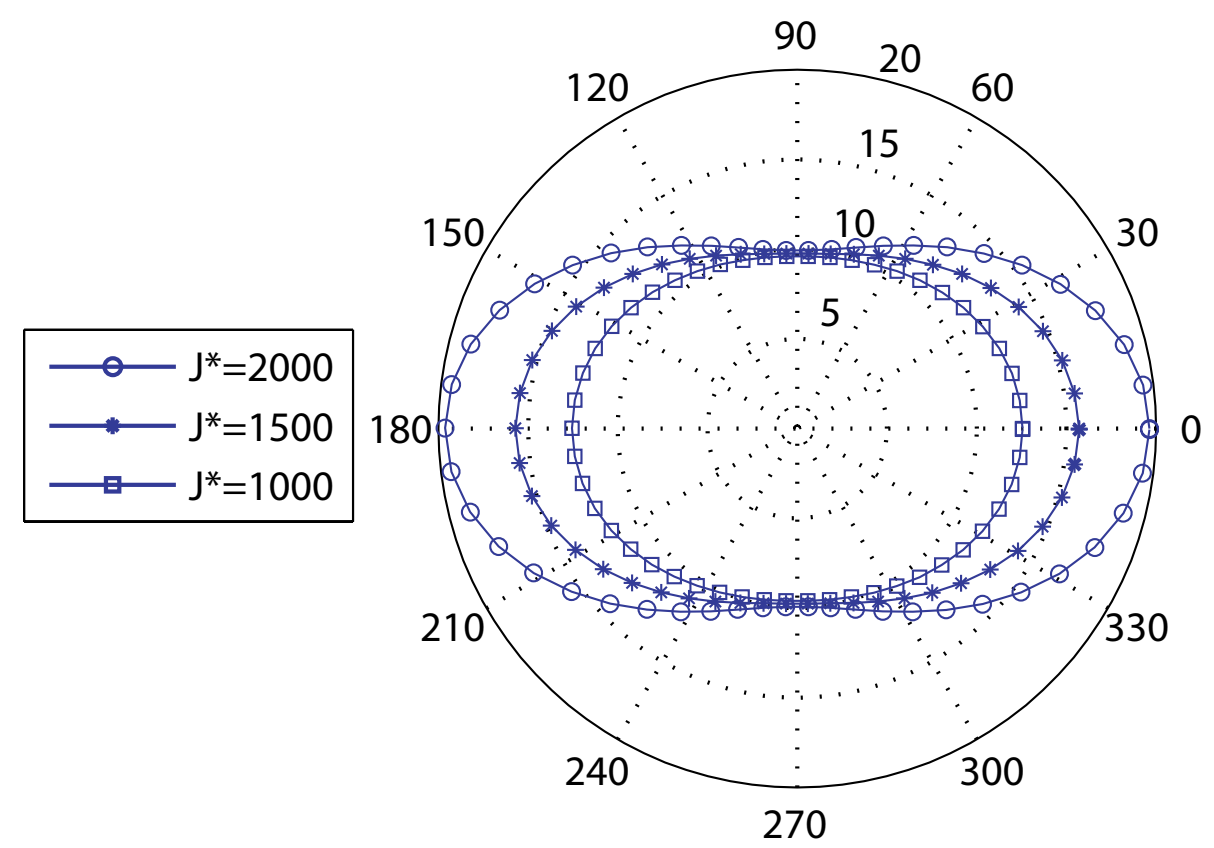

Fig. 6 Variations of the effective diffusivity with flow direction for $R / H=0.2$ 


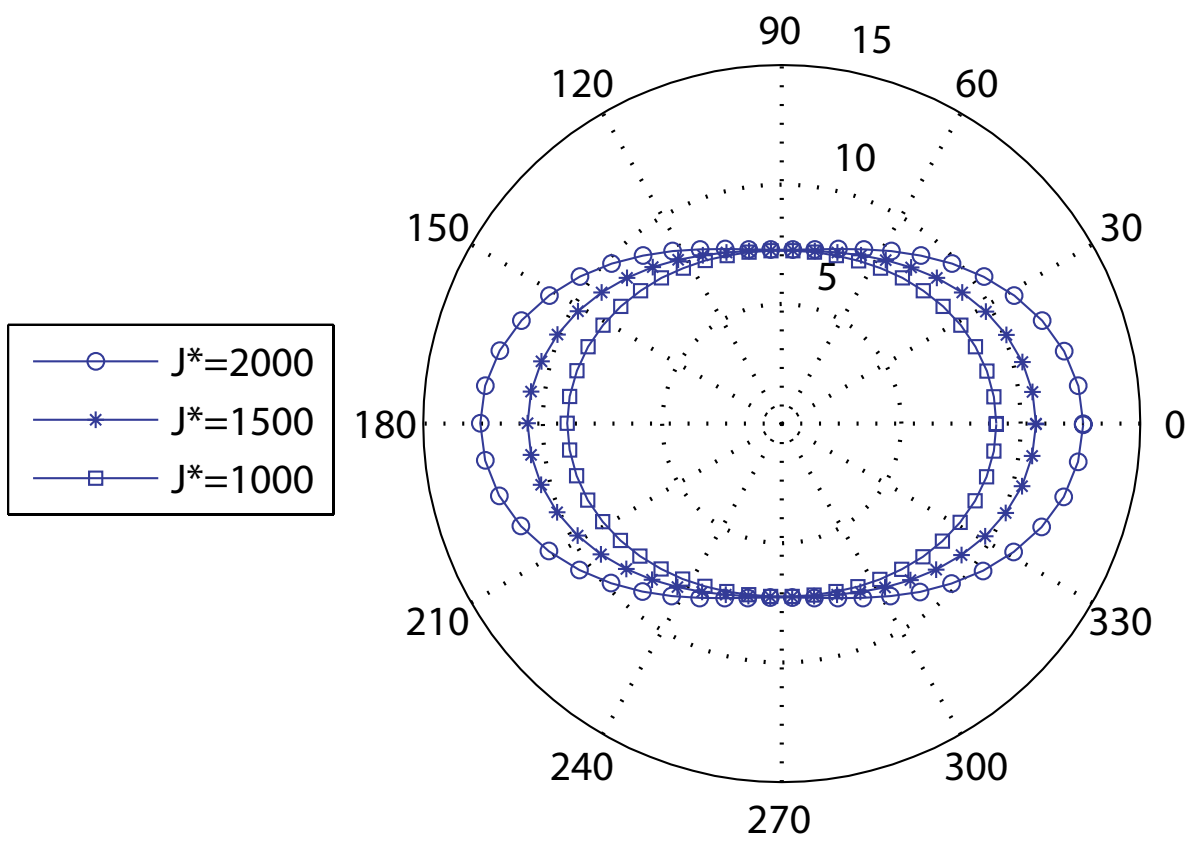

Fig. 7 Variations of the effective diffusivity with flow direction for $R / H=0.5$ 


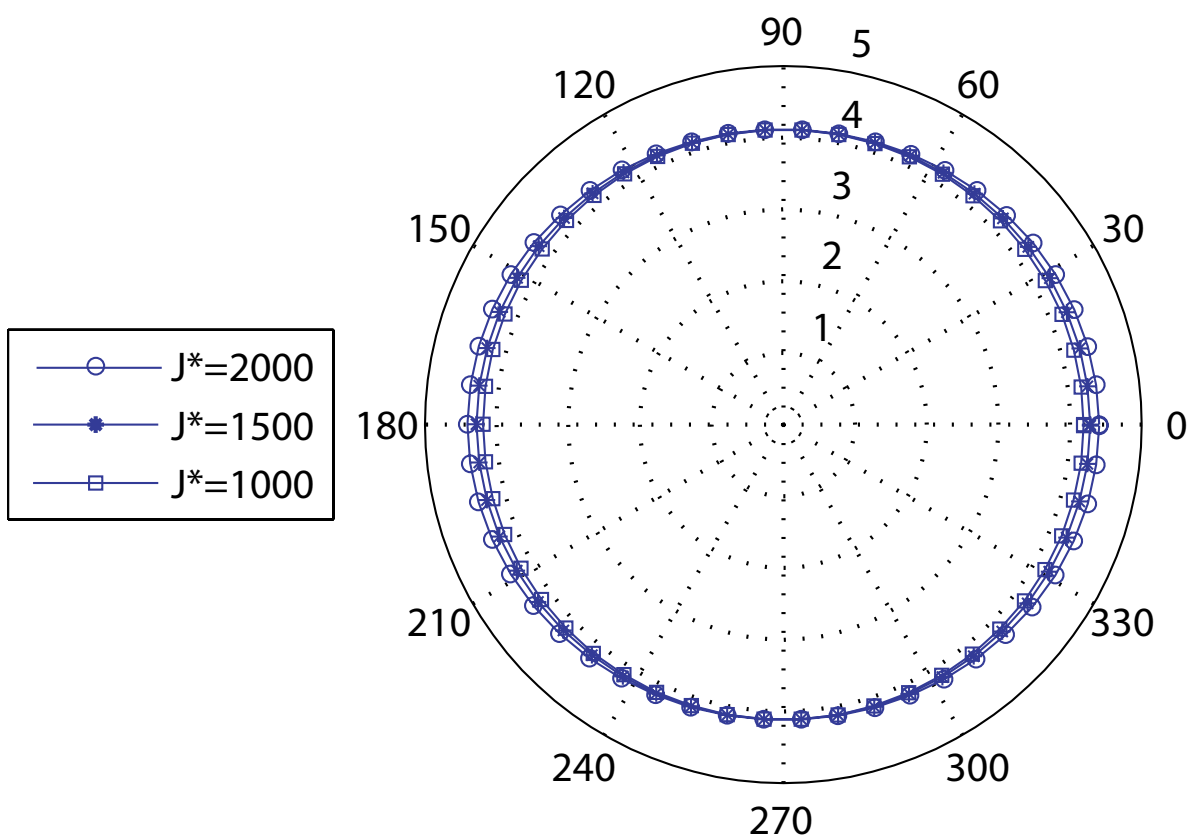

Fig. 8 Variations of the effective diffusivity with flow direction for $R / H=0.8$

4.3 Porous cell with randomly distributed and oriented fibers

In this section, we apply the method to $3 \mathrm{D}$ microstructures made up of interconnected random fibers. One example of such microstructure is provided on Fig. 9. To investigate the dispersion effect, the effective diffusivity is computed for various microstructure realization. The total number of fibers is $N=10$ with the radius $R / H=0.05$ (where $2 H$ is the dimension of the cubic unit cell). In order to obtain an interconnected porous medium, each cylinder intersects another by their respective axes but the orientation and position of the cylinders are chosen arbitrarily. The total volume of the solid is then uncontrolled and this also implies some dispersion in the porosity value.

The calculations are performed by taking 256 wave vectors along each space directions. For the Stokes flow problem, a stress based iterative scheme is used, this involving $6 \times 256 \times 256 \times 256 \sim 100.10^{6}$ DOFs. For the heat transfer problem, the method of resolution uses the polarization that is a vector, the total number of DOFs is $3 \times 256 \times 256 \times 256 \sim 50.10^{6}$. A contrast $k_{f} / k_{s}=10$ is taken as in previous examples. A gradient of pressure $J_{3}^{*}$ is prescribed in order to generate the flow in direction $O x_{3}$. The effective diffusivity $K_{33}$ is computed by imposing the gradient of temperature $E_{3}$. 


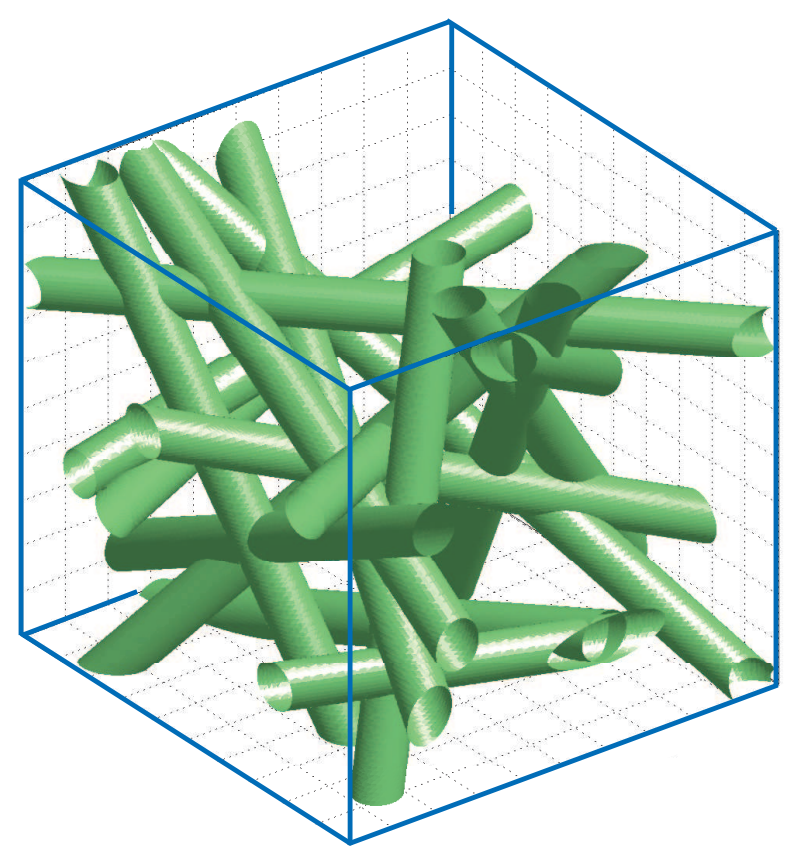

Fig. 9 Exemple of a unit cell of the porous medium with randomly distributed and oriented fibers

The results are presented on table 3 and on Figs. 10 and 11. The values of effective diffusivity $K_{33}$ (normalized by $k_{s}$ ) are provided on table 3 for three values of the pressure gradient. In the last column, are provided the values of the porosity. The values computed for the effective diffusivity are also given on Figs. 10 and 11 on which is also provided the cumulated average value of $K_{33} / k_{s}$. It is observed that the dispersion in the results are greatly increased with the value of the gradient of pressure. This effect will be attributed to both the low value of porosity and the fibers distribution influence. Indeed at higher values of gradient of pressure it has been already found in previous examples that the convection has a prominent influence on effective diffusivity. The dispersions in the results are then greatly increased between the different occurrences. A finely analysis of these uncertainty and on the choice of the representative volume element would be performed following $[26,27]$. This is not the main goal of the present application. 


\begin{tabular}{c|ccc|c}
$N^{0}$ & \multicolumn{3}{|c|}{$K_{33} / k_{s}$} & Volume fraction \\
\hline & $J_{3}^{*}=10$ & $J_{3}^{*}=1000$ & $J_{3}^{*}=2000$ & \\
1 & 1.1257 & 4.2988 & 12.5952 & 0.0311 \\
2 & 1.1087 & 4.1956 & 12.4108 & 0.0308 \\
3 & 1.1291 & 5.3233 & 17.2091 & 0.0314 \\
4 & 1.1305 & 2.6669 & 6.7024 & 0.0321 \\
5 & 1.1200 & 3.5004 & 9.6789 & 0.0316 \\
6 & 1.1114 & 4.4589 & 13.3691 & 0.0306 \\
7 & 1.1297 & 5.9579 & 19.3942 & 0.0314 \\
8 & 1.1138 & 3.0092 & 8.1635 & 0.0293 \\
9 & 1.1294 & 2.9117 & 8.0133 & 0.0319 \\
10 & 1.1442 & 2.4569 & 5.8313 & 0.0323
\end{tabular}

Table 3 Effective diffusivity for random fibers and for different occurrences. The number of fibers is $N=10$ and the contrast is $k_{f} / k_{s}=10$

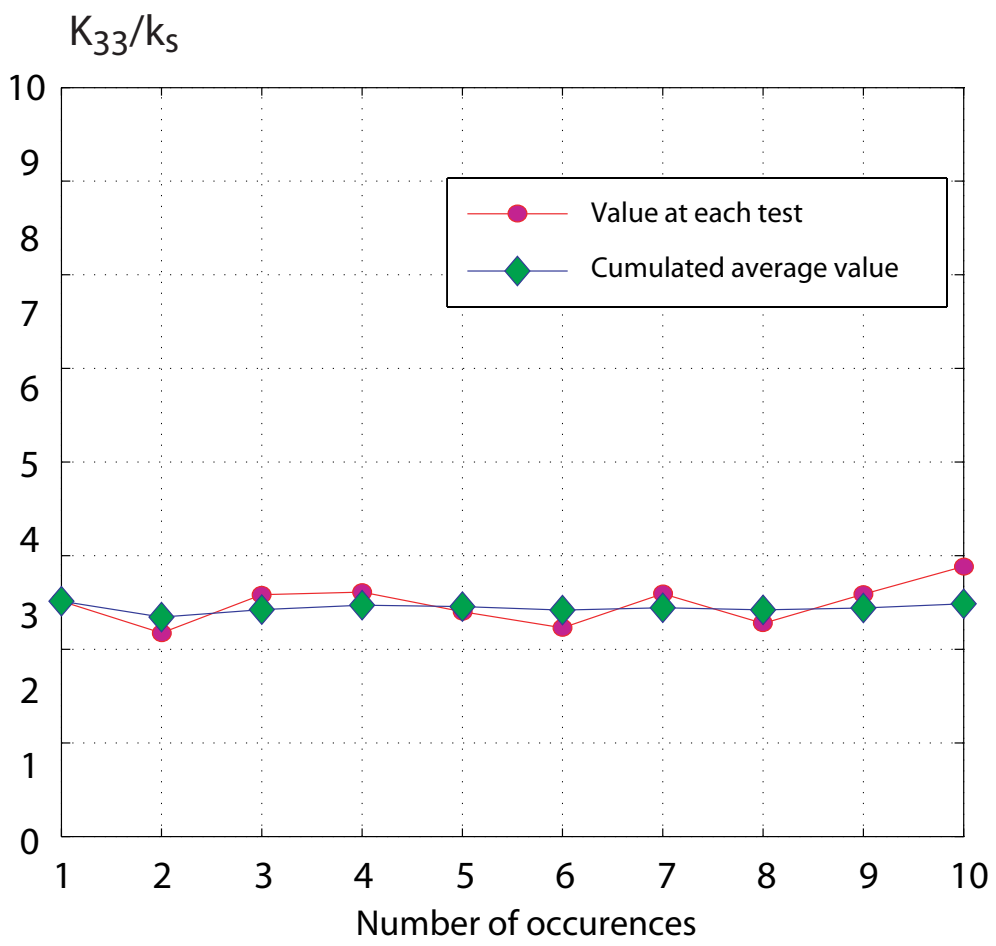

Fig. 10 Effective diffusivity for random fibers and for different occurrences (line with circles) and cumulated value of effective diffusivity (line with diamonds) for $J_{3}^{*}=10$. The number of fibers is $N=10$ and the contrast is $k_{f} / k_{s}=10$ 


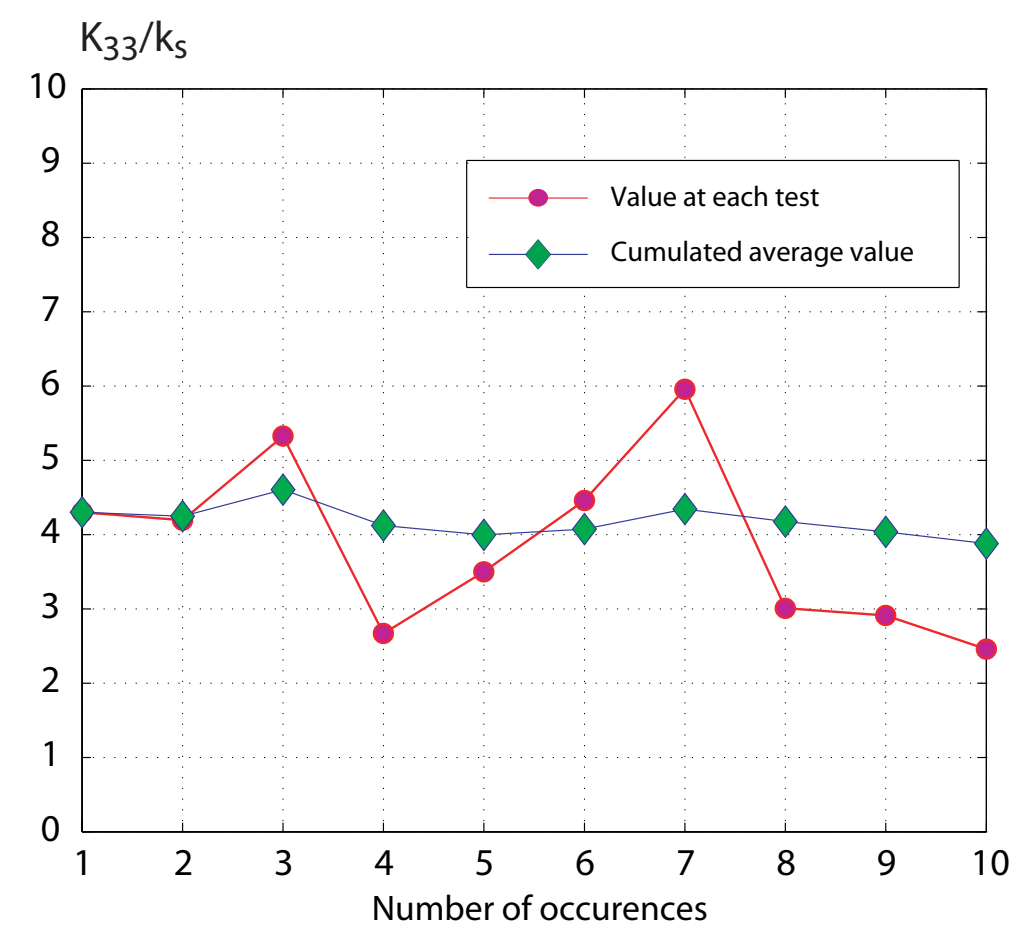

Fig. 11 Effective diffusivity for random fibers and for different occurrences (line with circles) and cumulated value of effective diffusivity (line with diamonds) for $J_{3}^{*}=1000$. The number of fibers is $N=10$ and the contrast is $k_{f} / k_{s}=10$

\section{Conclusion}

This paper provides a numerical method to compute the effective diffusivity of porous material saturated by a viscous fluid. The diffusivity is evaluated in the framework of periodic homogenization and consists in solving two unit cell problems successively: the Stokes flow problem under an applied pressure gradient, the heat transfer problem due to both conduction and convection. The resulting effective diffusivity then depends on the applied gradient of pressure. Two methods of resolution based on FFT and Green operators are provided to solve the heat transfer problem. A first simple one, is based on the gradient of temperature and uses the associated primal Green tensor. An accelerated scheme based on the polarisation and using both the primal and dual Green tensors is also proposed to increase the rate of convergence.

The accuracy and capacities of these two iterative schemes are evaluated through various $2 \mathrm{D}$ and $3 \mathrm{D}$ examples. First, the case of flows in parallel pores is considered as benchmark problem to compare the basic and the accelerated scheme and to check their accuracy with the exact solution. As expected, the accelerated scheme has been found to converge more rapidly and is only 
considered for the other applications. As a second application, the effective diffusivity is computed in the case of a regular array of cylinders. Particular attention is made on the role of the flow direction on the homogenized diffusivity. In a last example, we solve the problem for a complex 3D microstructure made up of interconnected cylinders of random orientations and which can mimic more realistic some fibers made porous materials.

\section{References}

1. J.-1. auriault, c. geindreau, c., boutin. Transp. Porous Med., 60(1)(89-108), 2005.

2. F.J. Alcocer, V. Kumar, and P. Singh. Permeability of periodic porous media. Phys. Rev. E., 59(1):711-714, 1999.

3. F.J. Alcocer and P. Singh. Permeability of periodic arrays of cylinders for viscoelastic flows. Phys. Fluids., 14(7):2578-2581, 2002.

4. G. Allaire and A.L. Raphael. Homogenization of a convection-diffusion model with reaction in a porous medium. C.R. Acad. Sci. Paris, Ser. I, 344:523-528, 2007.

5. A.A. Alshare, P.J. Strykowski, and T.W. Simon. Modeling of unsteady and steady fluid flow, heat transfer and dispersion in porous media using unit cell scale.

6. T. Arbogast and D. S. Brunson. A computatonal method for approximating a darcystokes system governing a vuggy porous medium. Computational Geosciences.

7. D.N. Arnold, F. Brezzi, and M. Fortin. A stable finite element for the stokes equations. Calcolo.

8. J.L. Auriault. Upscaling heterogeneous media by asymptotic expansions. J. Eng. Mech., $128(8): 817-822,2002$.

9. J.L. Auriault, C. Boutin, and C. Geindreau. Homogenization of coupled phenomena in heterogenous media. John Wiley \& Sons, 2010.

10. M. Bahloff, A. Mikelic, and M.F. Wheeler. Polynomial filtration law for low reynolds number flows through porous media. Transport in Porous Media, 81:36-60, 2010.

11. J. Barrere, J.P. Caltagirone, and O. Gipouloux. Détermination numérique de la perméabilité en milieu poreux périodique tridimensionnel. C. R. Acad. Sci. Paris, Sér. II., 310:347-352, 1990.

12. A.L. Berdichevsky and Z. Cai. Perform permeability predictions by self consistent method and finite element simulation. Polymer Composites, 14:132-143, 1993.

13. J.F. Bloch and J.L. Auriault. Heat transfer in nonsaturated porous media. modelling by homogenisation. Transport Porous Med., 30(3):301-321, 1998.

14. G. Bonnet. Effective properties of elastic periodic composite media with fibers. J. Mech. Phys. Solids, 55(5):881 - 899, 2007.

15. R.G. Carbonell and S. Whitaker. Heat and mass transfer in porous media. In Fundamentals of transport phenomena in porous media, pages 121-198. Springer, 1984.

16. J.L. Auriault E. Skjetne. New insights on steady, non-linear flow in porous media. Eur. J. Mech. B Fluids, 18(1):131-145, 1999.

17. D.A. Edwards, M. Shapiro, P. Bar Yoseph, and M. Shapira. The influence of reynolds number upon the apparent permeability of spatially periodic arrays of cylinders. Phys. Fluids A, 2:45-55, 1990.

18. P. Forchheimer. Wasserbewegung durch boden. Z. Ver. Deutsch. Ing, 45:1782-1788, 1901.

19. M. Fortin. Old and new finite element for icompressible flows. Int. J. Num. Meth. Fluids, 1:347-364, 1981.

20. T. Giorgi. Derivation of the forchheimer law via matched asymptotic expansions. Transport in Porous Media, 29:191-206, 1997.

21. Brinkman H.C. A calculation of the viscous force exerted by a flowing fluid on a dense swarm of particles. App. Sci. Res., 1:27-34, 1949.

22. Brinkman H.C. On the permeability of media consisting of closely packed porous particles. App. Sci. Res., 1:81-86, 1949. 
23. C. T. Hsu and P. Cheng. Thermal dispersion in a porous medium. Int. J. Heat Mass Transf., 33(8):1587-1597, 1990.

24. C.T. Hsu and P. Cheng. Thermal dispersion in a porous medium. Int. J. Heat Mass Transfer, 33(8):1587-1597, 1990.

25. Z. Idris, L. Orgeas, C. Geindreau, J.F. Bloch, and J.L. Auriault. Microstructural effects on the flow law of power law fluids through fibrous media. Model. Simul. Mat. Science Engrg., 12:317-330, 2004.

26. T. Kanit, S. Forest, I. Galliet, V. Mounoury, and D. Jeulin. Determination of the size of the representative volume element for random composites: statistical and numerical approach. Int. J. Solids Struct., 40(13-14):3647-3679, 2003.

27. P.S. Koutsourelakis. Stochastic upscaling in solid mechanics: an exercice in machine learning. J. Comput. Phys., 226(1):301-325, 2007.

28. F. Kuwahara, A. Nakayama, and H. Koyama. A numerical study of thermal dispersion in porous media. J. Heat. Transfer, 118(3):756-761, 2007.

29. H.-B. Ly, V. Monchiet, and D. Grande. Computation of permeability with fast fourier transform from 3d digital images of microstructures. Int. J. Num. Meth. Heat and Fluid Flow, 26(5):1328-1345, 2016.

30. I. Malinouskaya, V.V. Mourzenko, J.-F. Thovert, and P.M. Adler. Wave propagation through saturated porous media. Phys. Review E, 7:066302, 2008.

31. C.C. Mei and J.-L.Auriault. The effect of weak inertia on flow through a porous medium. J. Fluid Mech., 222:647Ü663, 1991.

32. J.-C. Michel, H. Moulinec, and P. Suquet. A computational scheme for linear and non-linear composites with arbitrary phase contrast. Int. J. Numer. Meth. Engrg., 52:139-160, 2001.

33. J.C. Michel, H. Moulinec, and P. Suquet. Effective properties of composite materials with periodic microstructure: a computational approach. Comput. Method Appl. M, 172(1-4):109 - 143, 1999.

34. G.W. Milton. The Theory of Composites. Cambridge University Press, 2002.

35. M. Moghari. A numerical study of non-equilibrium convective heat transfer in porous media.

36. V. Monchiet and G. Bonnet. A polarization-based fft iterative scheme for computing the effective properties of elastic composites with arbitrary contrast. Int. J. Numer. Meth. Eng., 89(11):1419-1436, 2012.

37. V. Monchiet and G. Bonnet. A polarization-based fast numerical method for computing the effective conductivity of composites. accepted for publication in Int. J. Numer. Meth. Heat Fluid Flow, 23(7):1256-1271, 2013.

38. V. Monchiet, G. Bonnet, and G. Lauriat. A fft-based method to compute the permeability induced by a stokes slip flow through a porous medium. CR Mecanique, 337(4):192-197, 2009.

39. H. Moulinec and P. Suquet. A fast numerical method for computing the linear and nonlinear mechanical properties of composites. C. R. Acad. Sci., 318(11):1417-1423, 1994.

40. H. Moulinec and P. Suquet. A numerical method for computing the overall response of nonlinear composites with complex microstructure. Comput. Meth. Appl. Mech. Engrg., 157:69-94, 1998.

41. C. Moyne. Two-equation model for a diffusive process in porous media using the volume averaging method with an unsteady-state closure. Adv. Water Resour., 20(2):63-76, 1997.

42. C. Moyne, S. Didierjean, H.P. Amaral Souto, and O.T. Da Silveira. Thermal dispersion in porous media: one-equation model. Int. J. Heat Mass Transfer, 43(20):3853-3867, 2000 .

43. T.-K. Nguyen, V. Monchiet, and G. Bonnet. A fourier based numerical method for computing the dynamic permeability of porous media. Eur. J. Mech. B/fluids.

44. T.K. Nguyen, V. Monchiet, and G. Bonnet. A fourier based numerical method for computing the dynamic permeability of periodic porous media. Eur. J. Mech. B, 2012.

45. M.H.J. Pedras and M.J.S. De Lemos. Thermal dispersion in porous media as a function of the solid-fluid conductivity ratio. Int. J. Heat Mass Transfer, 51(21):5359-5367, 2008 . 
46. E. Sanchez-Palencia and A. Zaoui. Homogenization Techniques for Composite Media. Springer Verlag, 1987.

47. T.L. Vu, G. Lauriat, and O. Manca. Forced convection of air through networks of square rods or cylinders embedded in microchannels. Microfluid. Nanofluid., pages 1-18, 2013.

48. S. Whitaker. Diffusion and dispersion in porous media. AIChE J., 13(3):420-427, 1967.

49. J.-C. Wodie and T. Levy. Correction non lineaire de la loi de darcy. C. R. Acad. Sci. Paris, Serie II, 312:157-161, 1991.

\section{A Poiseuille solution}

In the Poiseuille problem where the pressure gradient acting along the flow direction $\boldsymbol{J}=$ $J e_{1}$, the local velocity field of the fluid admits following parabolic form

$$
\begin{array}{ll}
v_{1}\left(x_{2}\right)=\frac{J}{2 \eta_{f}}\left[x_{2}^{2}-h^{2}\right] & \left|x_{2}\right| \leq h, \\
v_{1}\left(x_{2}\right)=0 & h \leq\left|x_{2}\right| \leq H
\end{array}
$$

In equation (63), $h$ is the pore height and $H$ the height of the unit cell (see figure 1). The macroscopic velocity field is:

$$
V_{1}=-\frac{J h^{3}}{3 H \eta_{f}}
$$

For the heat transfer problem, a macroscopic temperature gradient is prescribed in the same direction of the flow, i.e. $\boldsymbol{E}=E \boldsymbol{e}_{1}$. Adopting the following general form of temperature field in the fluid and solid phase

$$
T=E x_{1}+\psi\left(x_{2}\right),
$$

the differential equations for $\psi\left(x_{2}\right)$ in both the solid and the fluid phase are:

$$
\text { Fluid phase: } \begin{aligned}
k_{f} \psi^{\prime \prime}\left(x_{2}\right) & =\left[v_{1}\left(x_{2}\right)+k_{s} \frac{J^{*} h^{3}}{3 \eta_{f} H}\right] E \\
& =\frac{J^{*} k_{s} E}{H^{3}}\left[\frac{x_{2}^{2}}{2}-\frac{h^{2}}{2}+\frac{h^{3}}{3 H}\right]
\end{aligned}
$$

Solid phase: $k_{s} \psi^{\prime \prime}\left(x_{2}\right)=\frac{J^{*} k_{s} E h^{3}}{3 H^{4}}$

in which we have used the dimensionless pressure gradient:

$$
J^{*}=\frac{J H^{3}}{k_{s} \eta_{f}}
$$

After integration, we obtain (accounting for the symmetry of the problem):

$$
\begin{aligned}
& \text { Fluid phase: } \psi\left(x_{2}\right)=\frac{J^{*} k_{s} E}{H^{3} k_{f}}\left[\frac{x_{2}^{2} h^{2}}{4}-\frac{x_{2}^{4}}{24}-\frac{h^{3}}{6 H} x_{2}^{2}\right]+C_{1} \\
& \text { Solid phase: } \psi\left(x_{2}\right)=-\frac{J^{*} k_{s} E}{H^{3} k_{s}}\left[\frac{h^{3}}{6 H} x_{2}^{2}\right]+C_{2}+C_{3}\left|x_{2}\right|
\end{aligned}
$$

The integration introduces three constants $C_{1}, C_{2}$ and $C_{3}$ which have to be determined. The continuity of the temperature and the flux at the interface between the solid and the 
fluid (at $x_{2} \pm h$ ) leads to:

$$
\begin{aligned}
& C_{3}=-\frac{J^{*} h^{3} k_{s} E}{3 H^{3} k_{s}} \\
& C_{1}=C_{2}-\frac{J^{*} h^{4} k_{s} E}{24 H^{4}}\left[\frac{4 h-5 H}{k_{f}}+\frac{4(2 H-h)}{k_{s}}\right]
\end{aligned}
$$

The condition $\langle T\rangle_{V}=0$ yields to:

$$
C_{2}=\frac{J^{*} h^{3} k_{s} E}{H^{5}}\left[\frac{(5 h-6 H) h^{2}}{45 k_{f}}+\frac{2 H^{3}+3 H h^{2}-2 h^{3}}{18 k_{s}}\right]
$$

Note however that $C_{2}$ does not enter into the calculation of the homogenized permeability since it appears in (68) as a constant temperature.

The calculation of the permeability from relation (11) leads to:

$$
K_{11}=(1-f) k_{s}+f k_{f}+\left(J^{*}\right)^{2} k_{s}^{2} f^{6}\left[\frac{f\left(51-84 f+35 f^{2}\right)}{945 k_{f}}+\frac{(1-f)^{3}}{27 k_{s}}\right]
$$

Here only the component of $K_{11}$ is provided since a macroscopic gradient of pressure along the direction $O x_{1}$ has been prescribed to the unit cell. The component $K_{22}$ is not affected by the fluid flow and remains unchanged from the classic solution:

$$
K_{22}=\left[\frac{1-f}{k_{s}}+\frac{f}{k_{f}}\right]^{-1}
$$

